\title{
A Multi-Agent Approach to Combine Reasoning and Learning for an Ethical Behavior
}

\author{
Rémy Chaput \\ remy.chaput@univ-lyon1.fr \\ Univ Lyon, Université Lyon 1, LIRIS, \\ UMR5205, F-69622 \\ Lyon, France
}

\author{
Jérémy Duval \\ jeremy-duval1@hotmail.fr
}

France

\author{
Olivier Boissier \\ Olivier.Boissier@emse.fr \\ Mines Saint-Etienne, Univ Clermont \\ Auvergne, CNRS, UMR 6158 LIMOS, \\ Institut Henri Fayol, F - 42023 \\ Saint-Etienne, France
}

\author{
Mathieu Guillermin \\ mguillermin@univ-catholyon.fr \\ Lyon Catholic University - UCLy, \\ Sciences and Humanities Confluence \\ Research Center EA 1598, F-69288 \\ Lyon, France
}

\author{
Salima Hassas \\ salima.hassas@univ-lyon1.fr \\ Univ Lyon, Université Lyon 1, LIRIS, \\ UMR5205, F-69622 \\ Lyon, France
}

\begin{abstract}
The recent field of Machine Ethics is experiencing rapid growth to answer the societal need for Artificial Intelligence (AI) algorithms imbued with ethical considerations, such as benevolence toward human users and actors. Several approaches already exist for this purpose, mostly either by reasoning over a set of predefined ethical principles (Top-Down), or by learning new principles (Bottom-Up). While both methods have their own advantages and drawbacks, only few works have explored hybrid approaches, such as using symbolic rules to guide the learning process for instance, combining the advantages of each. This paper draws upon existing works to propose a novel hybrid method using symbolic judging agents to evaluate the ethics of learning agents' behaviors, and accordingly improve their ability to ethically behave in dynamic multi-agent environments. Multiple benefits ensue from this separation between judging and learning agents: agents can evolve (or be updated by human designers) separately, benefiting from co-construction processes; judging agents can act as accessible proxies for non-expert human stakeholders or regulators; and finally, multiple points of view (one per judging agent) can be adopted to judge the behavior of the same agent, which produces a richer feedback. Our proposed approach is applied to an energy distribution problem, in the context of a Smart Grid simulator, with continuous and multi-dimensional states and actions. The experiments and results show the ability of learning agents to correctly adapt their behaviors to comply with the judging agents' rules, including when rules evolve over time.
\end{abstract}

Permission to make digital or hard copies of part or all of this work for personal or classroom use is granted without fee provided that copies are not made or distributed for profit or commercial advantage and that copies bear this notice and the full citation on the first page. Copyrights for third-party components of this work must be honored.

For all other uses, contact the owner/author(s).

AIES '21, May 19-21, 2021, Virtual Event, USA

(C) 2021 Copyright held by the owner/author(s).

ACM ISBN 978-1-4503-8473-5/21/05.

https://doi.org/10.1145/3461702.3462515

\section{CCS CONCEPTS}

- Computing methodologies $\rightarrow$ Multi-agent systems; Multiagent reinforcement learning; Reasoning about belief and knowledge.

\section{KEYWORDS}

Ethics, Machine Ethics, Multi-Agent Learning, Reinforcement Learning, Hybrid Neural-Symbolic Learning, Ethical Judgment

\section{ACM Reference Format:}

Rémy Chaput, Jérémy Duval, Olivier Boissier, Mathieu Guillermin, and Salima Hassas. 2021. A Multi-Agent Approach to Combine Reasoning and Learning for an Ethical Behavior. In Proceedings of the 2021 AAAI/ACM Conference on AI,Ethics, and Society (AIES '21), May 19-21, 2021, Virtual Event,USA. ACM, New York, NY, USA, 11 pages. https://doi.org/10.1145/ 3461702.3462515

\section{INTRODUCTION}

As the number of applications using various Artificial Intelligence (AI) models increases, a societal and research debate arises about how to incorporate ethical capabilities into these AI models.

Moor points out that "As robots become increasingly autonomous, we will need to supply them with more and more ethical capabilities" [24]; he defines several kinds of ethical agents, such as the "ethical impact agent", which has an ethical impact on human lives, either beneficial or harmful. The fact that this definition corresponds to many actual applications is an incentive for AI researchers to develop more ethically-capable agents, such as "ethical implicit agents", which embed considerations to warrant ethical behavior or "ethical explicit agents", which can reason about ethics and handle conflicts between different ethical principles.

Building "ethical impact agents" while considering ethical implications, including when the agents are integrated into human society, relates to the "Ethics In Design", as defined by Dignum [19]. Another important aspect is the "Ethics By Design", which pertains to the production of an ethical behavior by the agent itself, through the integration of specific abilities, e.g., reasoning or learning moral rules. 
While this demand for ethical capabilities of autonomous artificial agents has been largely documented [28], the means to implement such competences are not clear. Some researchers advocate a symbolic formalization of moral rules, using a given type of logical expression, namely Top-Down approaches [1], while others prefer using Machine Learning (ML) to let an agent automatically learn behaviors, namely Bottom-Up approaches. Both approaches offer different advantages but also have several drawbacks, as we will detail later. Therefore, in this paper, we present a novel, hybrid approach to learn behaviors under the guidance of judgments originating from symbolic reasoning, using distinguished judging and learning agents. As Dignum points out, "By definition, hybrid approaches have the potential to exploit the positive aspects of the top-down and bottom-up approaches while avoiding their problems. As such, these may give a suitable way forward." [19].

Even though an artificial agent cannot qualify as a full ethical being, Dignum notes that "AI systems will make and are already making decisions that we would consider to have an ethical flavour if they were made by people" [19]. Along these same lines, we will use the expression "ethical behavior" to characterize actions made by an agent that would be considered ethical from a human point of view, according to a set of moral values. For example, an agent reasoning over the rule "Killing is immoral" and therefore deciding not to kill somebody, is exhibiting an ethical behavior according to our definition, although it is programmed to reason as such.

The paper is structured as follows: we first present the design principles that we retain from the existing literature and on which we build our proposal; this proposal is then detailed; experiments on a Smart Grid use case and their results demonstrate its feasibility; finally, the last section compares the approach to the literature, reviews the current limitations, and offers perspectives.

\section{BACKGROUND KNOWLEDGE}

In order to identify the design principles that underlie our approach, we first explore the literature on Machine Ethics, considering the integration of Ethics into AI. We then consider the field of Hybrid (Neuro-Symbolic) AI, to investigate how symbolic and learning methods in AI can be combined.

\subsection{Ethics and AI}

The study of Ethics inside AI models is a relatively new field, with some pioneering work around 2000 [2, 6, 31], and was given various names - Machine Morality, Friendly AI, Artificial Morality, Computational Ethics, etc. - the most established of which may be Machine Ethics, defined as a field "concerned with how machines behave toward human users and other machines. It aims to create a machine that's guided by an acceptable ethical principle or set of principles" [3].

It can first be noted that most of the previous studies on Machine Ethics focused on a single agent isolated in its environment [35]. We argue that it is important to consider multiple agents in interaction in a shared environment, as this is closer to what would happen in real-world situations. This also allows us to experiment with different ethics: what happens if agents are driven by different value systems, if an agent is amoral? Or worse, purely immoral? How do the other agents react, are they able to somehow compensate?
Machine Ethics implementations are traditionally divided into three categories [1], much like other applications in AI: Top-Down, Bottom-Up, Hybrid approaches. Top-Down approaches pertain to the formalization of one or more particular ethical principles, such as Kantian Categorical Imperative, using symbolic reasoning and logic programming. The advantages of such approaches include the ability to build upon experts' knowledge to implement the agent's rules, and an easier readability of these rules, e.g., for human regulators. Examples include the Ethical Governor [5], which inspired other works, for instance [13], which use a supervisor component on top of an agent, tasked with assessing the adequacy of the agent's proposed actions with a set of ethical considerations, implemented as pre-established rules, such as the Rules of Engagement or Laws of War. Another, different, approach is Ethicaa [15], in which multiple agents are given a set of ethical principles and a priority order on these principles, that they use to judge which actions are permissible and which one should be preferred. This judgment can be used on their own actions to select their next one, or on another agent's actions in order to compute a moral image of this agent, which serves as the basis for a trust process. However, these Top-Down approaches have a limited adaptability: any novel situation which does not fall under one of the specified rules cannot be correctly processed by the agent. In addition, the current societal acceptance may evolve over time, and the agents would need to adapt to these changes, even for already known situations.

Bottom-Up approaches tend to learn a behavior from a given dataset, such as labeled examples or experiences obtained through interactions with an environment; they are often able to generalize over this dataset and therefore adapt to novel situations. One of the best known works is perhaps GenEth [4], based on Ross' theory of prima facie duties, e.g., minimize harm to patient or maximize autonomy respect, which can be conflicting and that the agent must uphold. The agent must choose an action by reasoning on these duties and prioritizing them, depending on the current context. To do so, the authors propose to use Inductive Logic Programming (ILP) to learn a new ethical principle, in the form of a logical Horn clause, from a dataset of ethicists' decisions in several contexts. Another, fundamentally different work, proposes to use rewardshaping to learn an ethical behavior [33]: the authors assume that an average, non-task oriented, human behavior exhibits ethical considerations, and compute an ethical reward as the difference between the agent's behavior and the average human behavior to shape the task-specific reward. The final reward is defined as the sum of the task-specific reward, e.g., driving on a road, and the ethical, non-task oriented, reward, e.g., not running over people. A downside of such Bottom-Up approaches is that they are known to be harder to interpret. By extension, it would be difficult for a human regulator to understand the expected behavior; this is perhaps even more visible in the second example, where the expected behavior is concealed in the dataset of human behavior. Indeed, the ethical part of the reward is simply a mathematical formula of divergence; to determine if this behavior really is a good moral standard, one has no choice but to look directly at the possibly quite large dataset, which may be an almost unfeasible task in some complex use cases. The logical formula in the GenEth approach should be easier to interpret, but can still be quite hard to grasp should the formula consist of many atoms. Although they are learning methods and 
should therefore be able to generalize, the question of adaptation to evolving rules was not addressed in these works. As such, it is not known whether the agents would need to be completely retrained from scratch in case of a change in the expected behavior.

Finally, Hybrid approaches combine both Top-Down and BottomUp approaches, such that agents can learn ethical behaviors from experience while being guided by an existing ethical framework to force constraints on it and prevent it from diverging. Interested readers may refer to [19] and specifically the section 5.3 "Approaches to Ethical Reasoning by AI" for an in-depth discussion about the differences between Top-Down, Bottom-Up and Hybrid approaches, and what they entail.

In this paper, we defend a model consisting of an ethical agent using a Hybrid approach. We discuss the various means of Hybrid Neuro-Symbolic AI in the next section and how to integrate them in an ethical agent.

\subsection{Multi-Agent Hybrid Neuro-Symbolic AI for Ethics}

Works in AI are commonly divided into two categories: symbolic reasoning and neuron-alike learning (ML). However, Hybrid approaches try to combine advantages from both symbolic and neural AI. For example, a recent review of the Beliefs, Desires, Intentions (BDI) architecture lists several possibilities for integrating ML techniques, such as Computer Vision to help with the situation assessment of the environment [11]. The authors also argue that plans in a BDI agent are easy to explain to a human; it is also admitted that it is easier to introduce prior knowledge, e.g., from a non-developer domain expert, with symbolic rules.

Traditionally, Hybrid Neuro-Symbolic AI focuses on the integration of symbolic items into the neural (learning) module, so that the agent can learn symbolic concepts. For example, SOAR$\mathrm{RL}$ integrates the SOAR reasoning platform with Reinforcement Learning (RL) algorithms [25]; BDI-RL integrates RL into a BDI agent [12]. In the domain of Computer-Vision, CLEVRER recently outperformed previous state-of-the art algorithms by combining symbolic representations with a Deep Learning process [34].

Several works previously cited, which suggest adding a symbolic, often BDI, "Ethical Layer" atop an artificial agent $[5,13]$ are often labeled as Hybrid, in the sense that the agent corresponds to lowlevel interaction with the environment, while the Ethical layer corresponds to high-level reflection and planning. Yet, we argue that the agent does not truly learn from the Ethical Layer, but is rather constrained by it. Thus, the approach as a whole does not benefit from the learning's advantages, such as the ability to generalize.

To the best of our knowledge, the integration of a symbolic judgment to provide a numeric reward for neural agents has not been studied in Machine Ethics. Nevertheless, some researchers propose a Multi-Agent System in which judging agents are able to compute a judgment of others' actions, based on a set of beliefs over a situation [15].

Therefore, it seems more interesting to consider different agents, rather than integrating both reasoning and learning processes inside a single agent. By separating the hybrid method into a set of symbolic judging agents, and another set of neural learning agents, multiple advantages are introduced, e.g., the ability to evolve independently, leading to and benefiting from a process of rules coconstruction, as well as improved intelligibility of the moral rules.

\section{PROPOSED MODEL}

In this section, we describe our proposal, based on the following design principles retained from the literature:

- We focus on a Hybrid approach to combine the advantages of Top-Down reasoning and Bottom-Up learning approaches, such as the ability to generalize over unexpected situations, a better intelligibility of the expected behavior, and the possibility to introduce prior knowledge from domain experts.

- In order to allow for a co-construction process, judging and learning are separated into different agents, which can then evolve independently. For example, judging agents' rules can be updated by human designers while learning agents adapt their behavior to comply with these new rules, leading to a perspective of human-centered AI with a human-in-the-loop schema.

- Intelligibility of the expected behavior is increasingly recognized as important in all AI applications; to achieve this, the judgment process is implemented using explicit, symbolic, moral values and rules inside the judging agents. We argue that intelligibility is even more important within the context of Machine Ethics: human designers, stakeholders or regulators must be able to understand the judgment made, in order to assert compatibility with their set of ethical principles and to correct the rules otherwise.

- A richer feedback for learning agents can be obtained by combining a variety of points of view from multiple judging agents. Although it could technically be possible to implement multiple points of views inside a single agent, we find it clearer to distinguish each moral value as a different judging agent. This also paves the way for more complex interactions between the judging agents, such as negotiation processes, and offers a simple way to update rules by adding or removing judging agents.

- As mentioned above, it is important to evaluate the impact of several ethical impact agents in a shared environment; in this case, this corresponds to multiple learning agents.

- Finally, the expected behavior may evolve over time, as a result of our society's dynamics. Thus, agents need to be able to adapt to changing rules.

The architecture is first described from an abstract level, then we formalize the model as a Markov Game and explain the judging and learning agents' behaviors, as well as their interactions.

\subsection{Abstract Architecture}

In order to present the main pillars of our approach, let us consider a Multi-Agent System comprising both humans and artificial agents (learning agents and judging agents), represented in Figure 1. Some humans (designers) design a shared environment and autonomous agents so that they can carry out specific tasks; actions performed to solve these tasks will eventually impact the shared environment, and therefore impact humans (stakeholders and possibly designers). As discussed above, such agents are "ethical impact agents", 


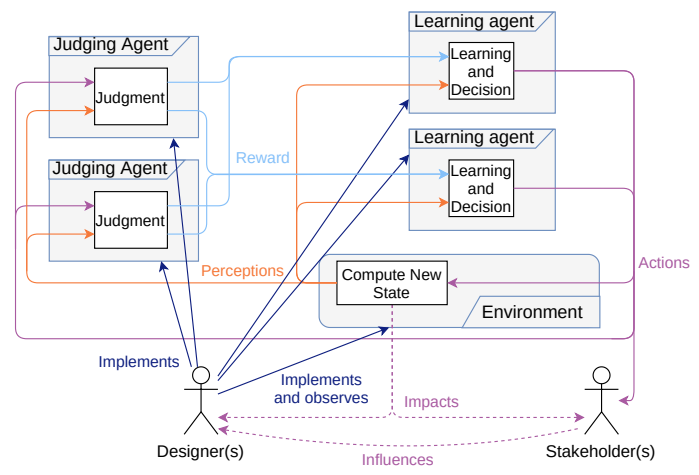

Figure 1: Abstract architecture of our approach, with human designers implementing rules to judge learners. Learners' actions modify a shared environment, which impacts humans.

according to Moor's definition [24]; the goal of designers is to embed ethical considerations in these agents, in order to produce a beneficial impact on stakeholders (in accordance with a given set of moral values selected by designers, either implicitly or explicitly).

We introduce specific judging agents, based on Ethicaa agents [15]; they are given a set of symbolic rules to reason over actions undertaken in the environment, and judge them. Rules can target consequences of actions (i.e., consequentialism) or actions themselves (i.e., deontological ethics).

Judging agents use these rules to compute a judgment (e.g., "moral", "immoral") and provide it as a feedback for learning agents. Learning agents learn a policy to solve the task, which may imply an ethical dilemma, by learning to select the correct action, according to this feedback, and actually performing it in the environment. This learned policy reflects the ethical considerations embedded in the symbolic rules.

Finally, human designers may observe the behavior of the learning agents and rectify the learners' decisions by adjusting the rules in the judging agents that generate feedback for the learning agents.

In this model, the source of ethical considerations we mentioned earlier is clearly visible: human designers select the set of rules which will be used to perform the judgment and thus direct the behavior of agents. Moreover, the set of moral values is explicit in this case, as the rules depend on them. An additional benefit is that, by clarifying such values and rules, we improve the transparency of the system so that human regulators, and more generally users, can asses whether they correspond to their own.

\subsection{Formal Model}

Let us consider the set $J$ of judging agents: each agent $j \in J$ is associated with a single moral value and has a set of rules to determine if a given action supports or defeats this moral value (e.g., Justice). In the second set $L$ of learning agents, each agent $l \in L$ learns a behavior and performs actions in the environment, based on feedback sent by all the judging agents $j \in J$. For example, considering $F_{l}$ the feedback received by learner $l$ and Judgment ${ }_{j}(l)$ the judgment performed by agent $j$ on $l$, we have the following: $\forall l \in L: F_{l}=\left\{\forall j \in J:\right.$ Judgment $\left._{j}(l)\right\}$.
We can conceptualize this learning model as a Stochastic (or Markovian) Game (SG), an extension of the well-known Markovian Decision Process (MDP) to multiple agents. Formally, this can be represented as a tuple $\left(S, P, A_{0}, \ldots, A_{n}, T, R_{0}, \ldots, R_{n}\right)$, where:

$S$ is the set of all possible states; in our case, we consider that states are represented by vectors of real values, or multidimensional continuous states. This allows for rich use cases.

$P$ is the set of all players (or agents), of size $n$; in our case, this corresponds to the set $L$ of Learners (remember that Judges do not act in the environment and are therefore not concerned).

$A_{l}$ is the set of all possible actions for a given agent $l$; similarly to the set $S$ of states, we will consider actions to be represented by vectors of real values, or "parameterized actions", where the vector components are the parameters of the action. We define $A$ as the set of all joint-actions, such that $A=A_{0} \times \ldots \times A_{n}$; in other terms, a joint-action is the combination of the different agents' actions at a given step.

$T$ is the transition probability function, noted $T: S \times A \times S \rightarrow$ $[0,1]$, i.e., $T\left(s, a, s^{\prime}\right)$ is the probability that performing action $a$ while in state $s$ will lead to state $s^{\prime}$.

$R_{l}$ is the reward function for a given agent $l$, noted $R_{l}: S \times$ $A_{l} \times S \rightarrow \mathbb{R}$, i.e., $R_{l}\left(s, a_{l}, s^{\prime}\right)$ is the reward for agent $l$ when performing action $a_{l}$ in state $s$ and resulting in state $s^{\prime}$.

MDPs and Stochastic Games can be solved with Reinforcement Learning (RL) [29], a method for learning a behavior, i.e., a strategy $\pi: S \times A \rightarrow[0,1]$ that will map each state-action pair to a probability, such that $\pi\left(s_{i}, a_{j}\right)$ is the probability of selecting action $a_{j}$ when in state $s_{i}$. For each of the learning agents, the goal of the RL algorithm is to learn the optimal strategy, so that the cumulative sum of received rewards is maximized.

Traditionally, the reward function is some sort of mathematical function that indicates whether the action was a good one, i.e., an objective to optimize. As mentioned earlier, we want to use the symbolic feedback computed by the judging agents. Thus, we need the reward function to transform this symbolic feedback into a single numeric value, by computing an aggregation of the judgments.

We first describe the learning agents, and the process by which they learn an optimal strategy $\pi$, leaving to one side the details of $R$ that we describe afterward.

\subsection{Learning Agents}

The learning agents are tasked with learning how to select an action in a given state so as to maximize the received reward over the time steps. We chose to use the Q-DSOM algorithm for its ability to handle the multi-dimensional and continuous features of states and actions [14]. This is thanks to a first component, named $D y$ namic Self-Organizing Map (DSOM) [27], derived from Kohonen's Self-Organizing Map [22]. DSOMs are 2D lattices of interconnected neurons, where each neuron is associated with a vector, or prototype, and learned over time by updating the vectors. Two different DSOMs are considered: a State-DSOM and an Action-DSOM, which learn to represent the state and action spaces, respectively.

The neurons are mapped to a second component, the well-known Q-Table [32], such that each neuron corresponds to a discrete state or action, i.e., a row or a column in the Q-Table. This Q-Table is 


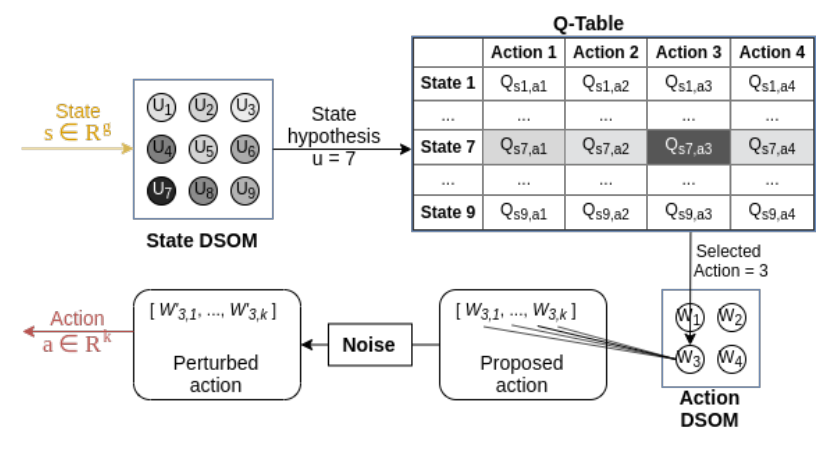

Figure 2: An example of a decision process: the agent receives a state, i.e., a vector of real values, which is compared to the State-DSOM. The 7th neuron has the closest vector prototype, so it is used as a state hypothesis. According to the $Q$-Table, having the best interest in the 7th state, the 3rd action is selected; the vector associated with the 3rd neuron in the Action-DSOM is perturbed by a random noise, which gives an action.

responsible for learning the interest, also called Q-Value, of a stateaction pair so that the agent is able to pick the best action for each given state.

The agents are therefore able to represent any multi-dimensional continuous state or action as a discrete identifier, and can use the Q-Table to determine the interest associated with this identifier, i.e., for this multi-dimensional continuous state or action.

Let us briefly explain the two main parts of the Q-DSOM algorithm, i.e., the decision process, used to select an action in a given state, and the learning process, used to update the agent's strategy so as to select a better action at the next decision step.

The decision process can be summarized as follows (see Figure 2 for an illustrated example):

(1) The agent receives a state $s$, i.e., a vector.

(2) $s$ is compared to the State-DSOM $U$; the agent assumes that the closest vector prototype $u$ is the "discrete state identifier" of $s$ (as the distance is minimal).

(3) $u$ corresponds to a neuron in the State-DSOM that is mapped to a row in the Q-Table; it is used to perform a lookup on the Q-Table and pick an action. While actions with the best interest are given a higher probability, we still give a nonzero probability to other actions, so as to try them and refine their estimated interest. The trade-off between exploiting the action that currently yields the best reward and exploring other actions (in case they yield even better rewards) is known as the exploitation-exploration dilemma, and is controlled by a standard Boltzmann exploration method. This process gives a "discrete action identifier" $w$.

(4) The agent selects the neuron $w$ from the Action-DSOM $W$ and uses its associated vector prototype as the proposed action.

(5) We noise the proposed action, in order to explore the action space and obtain the "perturbed action" $a$.

(6) The perturbed action $a$ is performed in the environment by the agent.

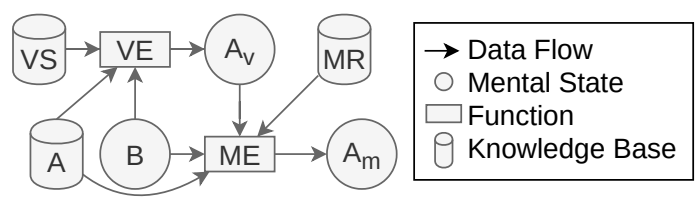

Figure 3: The judging agent architecture, adapted from Ethicaa [15].

After performing an action in the environment, the learner receives data to update its strategy; this learning process can be summarized as follows:

(1) The agent receives a new state $s^{\prime}$, i.e., a vector, and a reward $r$, a scalar value.

(2) If $r$ is better than the memorized interest of the state, i.e., the Q-Value, this means the action was a good one; so the Action-DSOM prototypes are updated toward the perturbed action $w^{\prime}$ (learning the action by trial-and-error).

(3) Then, the memorized interest of state-action pairs is updated, using the traditional Bellman equation.

(4) Finally, the State-DSOM is updated: prototypes are moved toward the previous state $s$. We know it is a "ground-truth" data, as it was received from the environment; the goal of the State-DSOM being to represent interesting states.

To summarize, the State- and Action-DSOMs learn to represent the state and action spaces. At each step, the agent computes a discrete state identifier by comparing the state vector to the prototypes in the State-DSOM and using the neuron with the closest prototype. The possible actions' interests are retrieved from the Q-Table row which corresponds to this state identifier; one of the actions' identifiers is picked. This action identifier corresponds to a neuron in the Action-DSOM, with an associated vector, which is noised in order to try a slightly different action and explore the action space. Performing the noised action in the environment leads to a new state and a reward, which are used to evaluate whether the picked action was a good one. The various components (StateDSOM, Action-DSOM and Q-Table) are updated to include this new data, so that the agent's strategy will improve over time.

\subsection{Judging Agents}

As we stated earlier, the judging agents are based on the Ethicaa agents [15], following a BDI architecture. Figure 3 shows the Moral Evaluation mechanism we use to produce a judgment.

At each step, judging agents generate beliefs (B) from their perceptions of the environment (the same as the learning agents) and from the perceived actions undertaken by these learning agents. Since learning agents handle actions as vectors of real values (i.e., multi-dimensional continuous actions) while judging agents handle actions as symbols (i.e., discrete actions), the judgment process treats each of the vector's components as a separate action. In other words, the judging agents receive a set of actions $\left\{\forall i \in[[1, k]]: a_{l, i}\right\}$, such that $a_{l}$ is a vector of $k$ components, and $a_{l, i}$ is the $i$-th component of the vector $a_{l}$, i.e., a real value.

The judging agent uses a fixed set of values and associated moral rules (VS and MR) to determine if each component of the perceived action defeats or supports the value. The Moral Evaluation (ME) 
uses the generated beliefs (B) to produce a moral valuation (Am), i.e., moral, immoral or neutral. Let us define the set of possible valuations $V=\{$ moral, immoral, neutral $\}$.

The feedback $F_{l}$ received by a learning agent $l$ is the set of judgments computed by all judging agents $j \in J$. We thus have $\forall l \in$ $L: F_{l}=\left\{\forall j \in J:\right.$ Judgment $\left._{j}(l)\right\}$. As described in this section, the judgment of a learning agent $l$ is the Moral Evaluation by a judging agent $j$ on every action component. Therefore, considering that $a_{l}$ is the action undertaken by agent $l$, and is described by a vector of $k$ parameters, we have Judgment ${ }_{j}(l)=\left\{i \in[[1, k]]: M E_{j}\left(B, a_{l, i}\right)\right\}$.

For example, where $k=3$, Judgment $j(l)=\{$ moral, neutral, immoral $\}$ means that the judging agent $j$ has evaluated the 1st component of the action undertaken by learning agent $l$ as moral, the 2 nd component as neutral, and the 3rd component as immoral.

\subsection{Judgment for Learning}

We have seen in previous sections that learning agents expect a numeric reward to leverage the Q-DSOM algorithm, whereas judging agents manipulate symbols. Similarly, judging agents expect to reason over beliefs, while learning agents use vectors of numbers as input. In this section, we describe the process that bridges the symbolic and numeric agents and achieves the necessary conversion.

At the end of each simulation step, after learning agents have chosen their actions, we consider various data, such as the list of agents, the actions per agent, the global properties representing the state of the environment, and the individual properties per agent; these are made available to the judging agents in the form of beliefs. These beliefs contain the aforementioned data as their parameters and trigger the judgment process.

Reusing the notations introduced earlier, the Moral Evaluation function $M E: B \times A \rightarrow V$ takes a set of beliefs and an action component, i.e., a real number, and returns a moral valuation; the Judgment function Judgment Ju $_{j} L \rightarrow V^{k}$ returns a list of valuations (of size $k$, which is the number of action components) for a given learning agent. Each judging agent computes a different judgment for each learning agent, such that the Feedback function $F: L \rightarrow$ $\left(V^{k}\right)^{|J|}$ returns a list of lists of valuations.

Finally, the reward function $R_{l}: S \times A_{l} \times S \rightarrow \mathbb{R}$ returns a real number. It can be noted that, as each judging agent computes a different judgment according to a specific moral value, there can be conflicts between the judgments. For example, the first judge may determine that the first component of the action is moral according to its own moral value, whereas a second judge may determine that the same first component of an action is immoral, according to its (different) moral value.

Many methods can be used to transform the symbolic valuations into a numeric reward; in these early stages, we propose the following solution. First, we transform the judgment of a single judging agent, i.e., a list of valuations, into a single number, by counting the number of moral valuations and dividing by the number of moral and immoral valuations, such that moral actions will tend toward 1 , while immoral actions will tend toward 0 . As a special case, if the list consists only of neutral valuations, we consider the action was neither good nor bad, and we set it to 0.5. Finally, the reward $r_{l}$ is simply the average of the rewards from each judging agent.
Note that we only use numbers between 0 and 1 , such that the mean does not produce any scaling issues. This assumption could be lifted by using one of the methods described in [20], in which several incomparable ethical theories are aggregated using, e.g., voting systems.

\section{EXPERIMENTS}

Let us consider a hypothetical micro-grid, in which electricity production is decentralized, instead of relying solely on the national network. The grid possesses a main source of electricity (e.g., a hydro-power station, or a windmill farm); the grid participants, or prosumers, besides consuming, can also produce a small amount of electricity (e.g., using solar panels). Considering the difficulty of storing large quantities of energy, and that production and demand can fluctuate over short periods, the prosumers of the same grid may exchange energy in order not to waste energy. Such local exchanges assume a form of cooperation to prevent unequal situations. Similarly, when the main source of the grid is overly solicited, prosumers must reduce their consumption, at least temporarily, thus reducing their comfort, in order to avoid blackout situations.

In the simulation, we consider a neighborhood of households, offices and schools: each building is represented by a learning agent in the Smart Grid, which has to learn how to consume and exchange energy in order to satisfy the comfort needs of the occupants, while considering the interests of other participants in the grid.

We consider that this simplified simulator and the opposition of interests between the different participants of the Smart Grid are both plausible enough and form an interesting framework to serve as a playground for ethical behaviors.

Relevant moral rules are presented in the following section. We then describe the various components of the simulator: the environment, the agents, possible actions, perceptions, and rewards.

\subsection{Relevance of Moral Rules and Values}

We reviewed the Smart Grid literature to find moral values [7, 18, 23]; these papers list the values that policy-makers (politicians, energy distribution companies, etc.) target when designing a decentralized energy system. Therefore, we adapted them to reflect the point of view of citizens, who are participating in a Smart Grid, and who must make decisions to allocate energy, in accordance with moral values. Indeed, our learning agents act as proxy for these prosumers and must uphold the very same moral values. We propose four moral values and associated rules as common references for all agents in our simulation:

MR1 - Security of Supply An action that allows a prosumer to improve its comfort is moral.

MR2 - Affordability An action that makes a prosumer pay too much money, w.r.t. the budget configured by the agent's user, is immoral.

MR3 - Inclusiveness An action that improves the equity of comforts between all prosumers is moral.

MR4 - Environmental Sustainability An action that prevents exchanges with the national grid is moral.

In his thesis, Bonnemains proposes to give a formal definition of an ethical dilemma [9]: a situation is an ethical dilemma if there is a choice between at least two possible actions and that each of the 


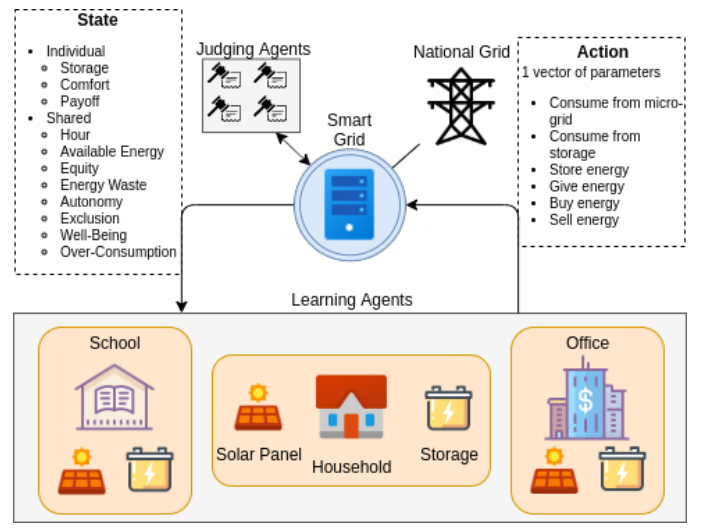

Figure 4: Schema of the Smart Grid simulator. A micro-grid is linked to the national grid, and contains multiple agents representing buildings: either a Household, an Office or a School. These agents receive a vector describing the state from the micro-grid, and perform an action with multiple parameters that allow them e.g., to consume energy.

alternatives implies a transgression of a given moral rule, either by its very nature or by the consequences of such an action, relating to a reference, such as a contextual norm, doctrine, moral value, principle or desire (we refer the interested readers to his thesis for the logic that led to this definition).

Based on the rules we propose, we can see that all exchanges with the national grid defeat the value associated with MR4. Moreover, an agent will try to improve its own comfort, according to rule MR1; but the amount of available energy is a finite quantity. Assuming that there is not enough energy to satisfy the comfort of all agents, consuming a given amount of energy will prevent another agent from improving its comfort, which results in a transgression of MR3. On the other hand, not consuming energy to allow another agent to be comfortable would be in accordance with MR3, but not with MR1.

Therefore, unless the quantity of available energy exceeds the total amount of energy required to satisfy each agent, no action can satisfy all moral rules; in other terms, every action implies a transgression of at least one moral rule, which classifies this simulation as an ethical dilemma, according to Bonnemains' definition.

The goal for our learning agents is, as the actions are continuous, to determine the right parameter so as to minimize regret. In other terms, the question can be, for example, "What amount of energy should I buy so as to minimize the transgression of MR4 while allowing myself to improve my comfort, which supports MR1?”.

\subsection{Simulator Description}

The simulator we used as our playground is illustrated in Figure 4; we detail its components below.

4.2.1 Learning Agents. The learning agents represent prosumers participating in the micro-grid; they receive perceptions from a shared environment, and perform actions such as consuming energy from the micro-grid or buying energy from the national grid.
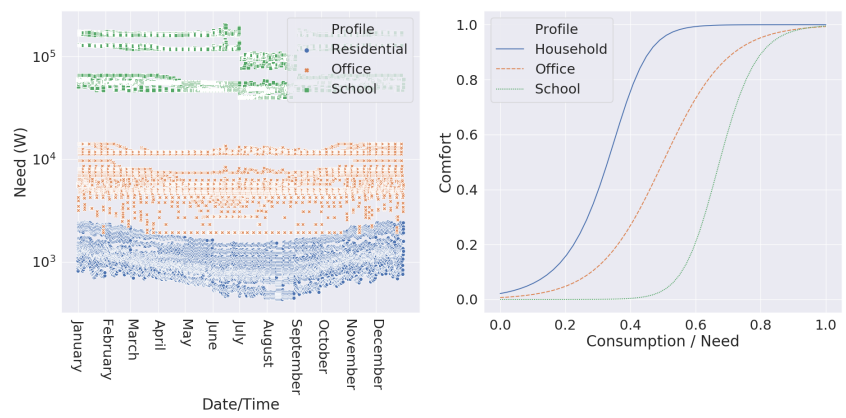

Figure 5: The needs and comforts for each agent profile.

To enrich the experiment, variety between the agents was introduced by creating three different profiles: Household, Office, and School. Each agent is associated with a profile, which determines: its consumption profile, i.e., the quantity of energy needed each hour; its comfort curve, i.e., the function that computes the comfort of the agent for a given consumption and need; its action range, e.g., the maximum amount of energy it can consume; and its storage capacity. For example, let us consider a Household agent and a School Agent; they need, respectively, $1007 \mathrm{~W}$ and $122784 \mathrm{~W}$ at a given time. The Household decides to consume $806 \mathrm{~W}$, whereas the School decides to consume $98227 \mathrm{~W}$; they both roughly consume $80 \%$ of their need. The Household has a flexible comfort curve, which means its comfort is evaluated to 0.99 , whereas the strict comfort curve of the School evaluates its comfort to 0.89 .

The original work [14] used a public dataset of energy consumption $^{1}$ as the source for the consumption profiles. Three buildings were selected: Residential, Small Office, and Primary School; each in the same city (Anchorage) in order to minimize the risk of possible bias between the profiles, e.g., not the same amount of heat needed. The dataset contains the hourly load profile, i.e., the amount of energy consumed by a typical building, for each hour over a year. While the authors decided to use an averaged profile, where hourly load was averaged over all days of the year, we retained the complete, annual profile. This introduces more difficulties for the learning agents, since the different seasons imply variations (e.g., we consume more energy in Winter than in Summer).

We handcrafted new comfort curves so that the strict curve corresponds to a low comfort when consumption is less than roughly $70 \%$ of the need; the flexible curve has a high comfort even for low consumption; and the neutral curve has a comfort of 0.5 when consumption is $50 \%$ of the need. The comfort curves and the energy needs per hour can be seen in Figure 5.

4.2.2 Actions. At each step, every learning agent performs an action, which is represented as a vector of parameters $a=[q, p, s, u, r, z] \in$ $\mathbb{R}^{6} . q$ is the quantity of energy consumed from the micro-grid. If the grid does not have enough energy, it is automatically bought from the national grid to prevent a blackout, but the quantity is considered as over-consumed. The agent can also consume a quantity $p$ from its personal storage, and conversely, give a quantity $s$

\footnotetext{
${ }^{1}$ https://openei.org/datasets/dataset/commercial-and-residential-hourly-loadprofiles-for-all-tmy3-locations-in-the-united-states
} 
from its storage to the micro-grid. $u$ is the quantity of energy the agent takes from the micro-grid for its personal storage, to be used later. The agent also interacts with the national grid, by buying a quantity $r$ of energy to store in its personal storage, and by selling a quantity $z$ from its storage to the national grid.

4.2.3 Perceptions. In order to take a decision, the agent must be informed of the current state of the environment; this state is represented by a vector of real values. We introduce a few notations to simplify their description: Comforts is the set of agents' comforts; Taken $_{i}=q_{i}+u_{i}$ is the quantity of energy that agent $i$ took from the micro-grid; Transactions $s_{i}=r_{i}+z_{i}$ is the amount of energy exchanged by agent $i$ with the national grid. The perceptions include common data shared by all agents:

- Hour: The current hour of the environment. Computed as (Stepmod24)/24 so each time step represents an hour.

- Available energy: The quantity of energy initially available in the micro-grid.

- Equity: Statistical measure of dispersion of the Comforts set, using the Hoover index, which is a well-known economical indicator of inequality. Computed as $1-$ Hoover (Comforts), such that a value close to 0 indicates an unequal distribution (only one agent has a high comfort), and a value close to 1 indicates an equal distribution (all agents have the same comfort).

- Energy Waste: Available energy that was not taken by the agents.

- Autonomy: The absence of transactions with the national grid.

- Well-Being: The median of the agent's comforts.

- Exclusion: The proportion of agents that have a comfort less than $50 \%$ of the median comfort.

- Over-consumption: The proportion of energy that was taken by the agents but not initially available.

The agents also perceive additional details about themselves, which other agents cannot access:

- Personal storage: The ratio of available quantity of energy in the agent's personal battery, over the capacity of this battery

- Comfort: The comfort of the agent at the previous step.

- Payoff: The quantity of money the agent gained (or lost) through its transactions with the national grid (by selling or buying energy).

4.2.4 Rewards. As described in our model, the rewards are computed as judgment by the judging agents. They are implemented in Jason [10] (an extension of AgentSpeak [26]) and use the JaCaMo platform [8], which itself is implemented in Java. In order to solve the communication problem between the learning agents, implemented in Python, and the Java part, we decided to use REST messages, inspired by a proof-of-concept framework for learning in JaCaMo [12].

We implemented four judging agents, one for each of the moral values introduced; the agents judge each component of the actions (e.g., consume from micro-grid, consume from storage, buy from national grid, etc.), while having access to the Perceptions of each agent.
Judging agents contain rules in a pseudo-Prolog language, such as valueSupport (give_energy $(X)$ ) :- $X>0$, which means that the action of giving a quantity $X$ of energy supports the associated value (in this case, Environmental Sustainability) only if the quantity is greater than zero (non-null). Similarly, valueDefeat rules determine if an action defeats the value (the complete list of moral rules can be seen in the supplementary materials).

\section{RESULTS}

We devised multiple sets of simulations based on several parameters:

- Number of learning agents in the environment: "Small" simulations contain 20 Households, 5 Offices, and 1 School, while "Medium" simulations contain 80 Households, 19 Offices, and 1 School. This allows us to assess the scalability of our approach.

- Consumption profile: "Annually" simulations use the complete dataset of hourly energy consumption, for every day of the year, including seasonal variations and the like, whereas "Daily" simulations use an averaged dataset.

- Judges' configuration: we propose seven scenarios, four that include a single judging agent (named "mono-values"), one in which judging agents are activated one by one at different time steps ("Incremental"), one in which judging agents are initially all activated and are deactivated one by one at different time steps ("Decremental"), and one "Default" scenario where all four agents are activated the whole time. This variety of scenarios allows us to compare the presence and absence of each moral rule, the impact on other rules, and the ability of learning agents to adapt when the rules evolve over time, either by adding rules or removing them.

By combining these parameters, we obtained a total of $2 \times 2 \times 7=28$ sets of simulations. All sets were run 20 times on 10,000 steps.

A comparison of the "Small" and "Medium" experiments did not show a true difference in means (T-Test, $\mathrm{p}$-value $=0.83$ ), hinting at the scalability of our approach, although the execution time was naturally much longer.

We will focus on "Default" and "Incremental" scenarios as they are the most relevant; "mono-values" are useful as some sort of control scenarios to compare the effect of a moral value on the agent's behaviors when isolated and when aggregated with other values. The "Decremental" scenario shows the ability to remove rules but is not as interesting as the ability to add rules (the "Incremental" scenario). We also focus on the "Annually" consumption profile, as it contains seasonal variations, i.e., more difficulty for our learning agents.

Figure 6 shows that the average reward increased over the time steps, meaning that our proposed method is effective and that the learning agents are able to learn to comply with the given rules. Yet, there is a drop in rewards at the end of the simulation, mostly due to the "Inclusiveness" reward, which seems harder to learn. It is not clear whether this is a problem with the learning algorithm or with our implementation of moral rules. We can remark that the "Inclusiveness" value is the one with the greatest number of implemented rules; perhaps this can be linked with its difficulty to be learned. 


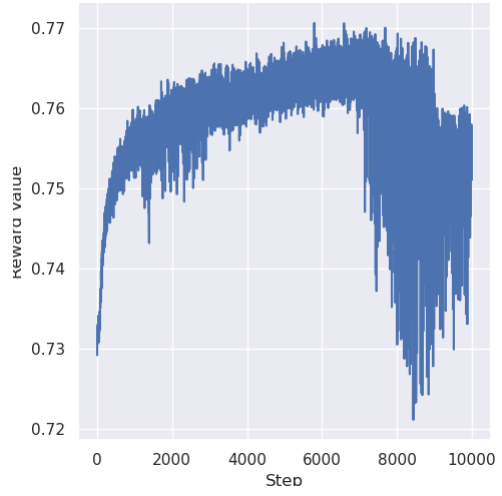

Figure 6: Global rewards, i.e., the mean of all learning agents' rewards on each time step, over the "Small - Annually - Default" runs.

Moreover, Figure 7 shows that agents were able to learn the "Environmental Sustainability" value in particular when given the aggregated reward, although the variation was not as important as when they were only given this specific value as reward. However, the "Inclusiveness" value seemed harder to learn, in particular at the end of the experiment, where the curve begins to drop. Interestingly, the comparison between "Default" and "Incremental" shows that adding the Judges one by one seems to mitigate this negative impact on "Inclusiveness". Agents are still able to learn "Environmental Sustainability", and perform slightly better on "Inclusiveness".

According to these figures, School agents had the most variations in their rewards, whereas Households and Offices had a steadier increase. This is not surprising, as the School agent has the greatest range of action, and thus a greater impact on the shared environment.

\section{DISCUSSION}

As a reminder, our contribution is the proposition of a novel method to learn "ethical behaviors", i.e., behaviors that exhibit one or more moral values and that would be considered ethical from a human point of view, using symbolic judgments as sources of rewards for learning agents in a multi-agent system. We evaluated this approach on an energy distribution problem, in a simulated SmartGrid context. The experiments can be improved still further but serve as a proof-of-concept that demonstrates the interest of this work.

With regard to the existing literature, this approach offers several advantages.

First of all, it is important to note that the current societal common acceptance of ethics can evolve over time. Thus, approaches that target Ethics By Design must consider the capability of the system to adapt to changing rules. Even among papers focusing on learning, this aspect was not extensively studied. In this paper, we showed thanks to the "Incremental" and "Decremental" experiments scenarios, the ability of our agents to adapt when adding or removing rules.

This is particularly striking when compared with the rewardshaping work discussed earlier [33]: if the example behavior is no

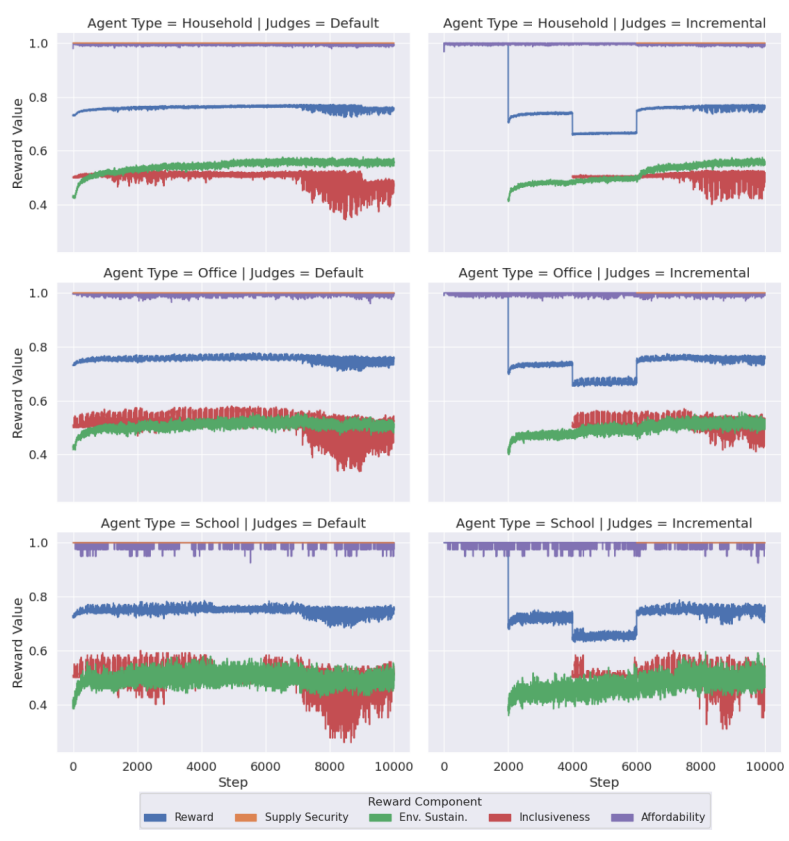

Figure 7: Comparison of individual rewards for each learning agent, over the time steps, averaged over "Small - Annually - Default" and "Small - Annually - Incremental" runs, and over agents with the same profile. The "Reward" curve is the mean of all four components, as described in the model.

longer in line with the expected behavior, they would need to recreate a new dataset and probably to train the agent from scratch. In our case, we can simply add or remove relevant rules. However, one advantage of their approach is that they assume a non-task oriented ethical reward, whereas we implement domain-specific moral rules. A possibility could be to implement general-purpose moral rules. However, this would require first and foremost the existence of such rules; a possible source of inspiration may be the numerous guidelines on Ethical or Responsible AI [28].

We also point out that previous use cases for "ethical agents" (be they learners or reasoners) were limited to discrete actions, from textbook ethical dilemmas, such as the best known Trolley Dilemma [30], to more realistic situations, such as an eldercare robot [4], soldier robots [5], and autonomous asset management [16]. These works are important both for understanding how to program "ethical agents" and because such situations are likely to happen. Nonetheless, we argue that applications requiring finer actions are numerous. It is therefore important to propose and experiment on such challenging environments, using continuous actions, such as the Smart Grid simulator we used here.

Nevertheless, a few approaches can use formal verification methods to assess compliance with the moral rules in any identified situation, due to a large extent to their symbolic BDI architecture [13]. Even though we also use symbolic BDI agents to provide the moral rules, the introduction of neural learning agents impairs this capability. However, there are some existing works that try to apply formal verification on RL algorithms [17, 21]. This is an important 
point that, in our opinion, should be focused upon in future works, as there may be some use cases where guarantees are needed.

An additional benefit ${ }^{2}$ of $\mathrm{RL}$ agents is that they learn a policy based on the expected reward, thus considering sequences of stateaction pairs, whereas most Top-Down approaches only consider the next action. One could imagine a scenario where a Top-Down agent would select the best action for a given state, w.r.t. the moral rules, but ends up in a new state where only actions that violate moral rules are available. In comparison, RL agents would both consider the immediate next action and the potential resulting states. This alleviates the designers' work, who only have to specify rules to judge the action, instead of considering a sequence.

We have seen that this work targets ethics by design considerations, but there are also side implications for the potential integration of such a solution into society. Indeed, we can observe at least one positive impact and one negative. First, the use of symbolic rules is said to be easier to trace than a mathematical (numeric) function, and therefore is supposed to be more understandable. For example, compare the following simple reasoning, along the rule of climate change mitigation: "Fighting climate change is moral. Using nuclear energy instead of coal helps fight climate change. Therefore, using nuclear energy is moral." with the pseudo-reward function $R=($ nuclear - coal $) / 100$. However, intelligibility was not the main objective of this work and was not focused on in our experiments. We believe that the intelligibility of the reward process is crucial, in particular for human supervision, either from the original designers of the system, or by third-party regulators. As such, this is an important point to consider and improve in future works.

On the other hand, judgments might need extensive data on the learning agents, e.g., their actions, their perceptions, etc., which impedes their privacy. It may be possible to limit the exchanged data by offering limited judgments, or even anonymize the data so that judges cannot identify the agents. In this paper, we simply assumed that data was freely available to the judging agents.

Our approach still has several limitations:

- The moral rules we used for evaluation are somewhat simple, and could perhaps be replaced with numeric functions, thus reducing the interest of the hybrid system. While this was a necessary step for proving the feasibility of such hybrid approaches, it would be interesting to extend the judging agents and their moral rules to enable them to judge more complex situations.

- The method for producing a numeric reward from a set of symbolic judgments, by mapping the symbols to numbers and taking the average makes it easy to deal with conflicting rules. However, other methods are possible. In particular, taking advantage of symbolic reasoning, it could be interesting to add a negotiation or argumentation mechanism between the judging agents to solve such conflicts, such that a priority between rules can be established in a given context. For example, consider the case where an important building (e.g., school or hospital) is crucially in need of energy; the rule that forbids buying energy from the national grid, according

\footnotetext{
${ }^{2}$ We thank one of the anonymous reviewers for pointing out this.
}

to the Environmental Sustainability value, can perhaps be set aside in this context.

\section{ACKNOWLEDGMENTS}

This work is funded by the French Région Auvergne Rhônes-Alpes (AURA), as part of the Ethics.AI project (Pack Ambition Recherche). We gratefully acknowledge support from the CNRS/IN2P3 Computing Center (Lyon - France) for providing computing and dataprocessing resources needed for this work

\section{REFERENCES}

[1] Colin Allen, Iva Smit, and Wendell Wallach. 2005. Artificial Morality: Top-down, Bottom-up, and Hybrid Approaches. Ethics and Information Technology 7, 3 (Sept. 2005), 149-155. https://doi.org/10.1007/s10676-006-0004-4

[2] Colin Allen, Gary Varner, and Jason Zinser. 2000. Prolegomena to Any Future Artificial Moral Agent. F. Exp. Theor. Artif. Intell. 12 (July 2000), 251-261. https: //doi.org/10.1080/09528130050111428

[3] Michael Anderson and Susan Leigh Anderson. 2006. Guest editors' introduction: Machine ethics. IEEE Intelligent Systems 21, 4 (2006), 10-11.

[4] Michael Anderson, Susan Leigh Anderson, and Vincent Berenz. 2019. A ValueDriven Eldercare Robot: Virtual and Physical Instantiations of a Case-Supported Principle-Based Behavior Paradigm. Proc. IEEE 107, 3 (2019), 526-540. https: //doi.org/10.1109/JPROC.2018.2840045

[5] Ronald C Arkin, Patrick D Ulam, and Brittany Duncan. 2009. An ethical governor for constraining lethal action in an autonomous system. Technical Report. Georgia Institute of Technology.

[6] Kevin D. Ashley and Bruce M. McLaren. 1995. Reasoning with Reasons in Case-Based Comparisons. In Case-Based Reasoning Research and Development, First International Conference, ICCBR-95, Sesimbra, Portugal, October 23-26, 1995, Proceedings (Lecture Notes in Computer Science, Vol. 1010), Manuela M. Veloso and Agnar Aamodt (Eds.). Springer, 133-144. https://doi.org/10.1007/3-540-605983_13

[7] Anne Boijmans. 2019. The Acceptability of Decentralized Energy Systems. Master's thesis. Delft University of Technology.

[8] Olivier Boissier, Rafael Bordini, Fred Hübner, Jomi, and Alessandro Ricci. 2020. Multi-Agent Oriented Programming: Programming Multi-Agent Systems Using faCaMo. The MIT Press.

[9] Vincent Bonnemains. 2019. Formal ethical reasoning and dilemma identification in a human-artificial agent system. (Formalisation du raisonnement éthique et de l'identification de dilemmes dans un système humain - agent artificiel). Ph.D. Dissertation. Institut supérieur de l'aéronautique et de l'espace, Toulouse, France. https://tel.archives- ouvertes.fr/tel-02890595

[10] Rafael H Bordini, Jomi Fred Hübner, and Michael Wooldridge. 2007. Programming multi-agent systems in AgentSpeak using Jason. Vol. 8. John Wiley \& Sons.

[11] Rafael H Bordini, Amal El Fallah Seghrouchni, Koen Hindriks, Brian Logan, and Alessandro Ricci. 2020. Agent programming in the cognitive era. Autonomous Agents and Multi-Agent Systems 34 (2020).

[12] Michael Bosello and Alessandro Ricci. 2020. From Programming Agents to Educating Agents - A Jason-Based Framework for Integrating Learning in the Development of Cognitive Agents. In Engineering Multi-Agent Systems, Louise A. Dennis, Rafael H. Bordini, and Yves Lespérance (Eds.). Springer International Publishing, Cham, 175-194.

[13] Paul Bremner, Louise A Dennis, Michael Fisher, and Alan F Winfield. 2019. On proactive, transparent, and verifiable ethical reasoning for robots. Proc. IEEE 107, 3 (2019), 541-561.

[14] Rémy Chaput, Olivier Boissier, Mathieu Guillermin, and Salima Hassas. 2020. Apprentissage adaptatif de comportements éthiques. In Architectures multi-agents pour la simulation de systeèmes complexes - Vingt-huitième journées francophones sur les systèmes multi-agents, FFSMA 2020, Angers, France, Fune 29 - July 3, 2020, Nicolas Sabouret (Ed.). Cépaduès.

[15] Nicolas Cointe, Grégory Bonnet, and Olivier Boissier. 2016. Ethical Judgment of Agents' Behaviors in Multi-Agent Systems. In Proceedings of the 2016 International Conference on Autonomous Agents \& Multiagent Systems (AAMAS '16). International Foundation for Autonomous Agents and Multiagent Systems, Singapore, Singapore, 1106-1114.

[16] Nicolas Cointe, Grégory Bonnet, and Olivier Boissier. 2016. Multi-Agent Based Ethical Asset Management. In Proceedings of the 1st Workshop on Ethics in the Design of Intelligent Agents, The Hague, The Netherlands, August 30, 2016 (CEUR Workshop Proceedings, Vol. 1668), Grégory Bonnet, Maaike Harbers, Koen V. Hindriks, Mike Katell, and Catherine Tessier (Eds.). CEUR-WS.org, 52-57. http: //ceur-ws.org/Vol-1668/paper9.pdf 
[17] Davide Corsi, Enrico Marchesini, Alessandro Farinelli, and Paolo Fiorini. 2020 Formal Verification for Safe Deep Reinforcement Learning in Trajectory Generation. In Fourth IEEE International Conference on Robotic Computing, IRC 2020 Taichung, Taiwan, November 9-11, 2020. IEEE, 352-359. https://doi.org/10.1109/ IRC.2020.00062

[18] TE de Wildt, EJL Chappin, G van de Kaa, PM Herder, and IR van de Poel. 2019 Conflicting values in the smart electricity grid a comprehensive overview. Renewable and Sustainable Energy Reviews 111 (2019), 184-196.

[19] Virginia Dignum. 2019. Responsible Artificial Intelligence: How to Develop and Use AI in a Responsible Way. Springer Nature.

[20] Adrien Ecoffet and Joel Lehman. 2020. Reinforcement Learning Under Moral Uncertainty. CoRR abs/2006.04734 (2020). arXiv:2006.04734

[21] Nathan Fulton and André Platzer. 2018. Safe Reinforcement Learning via Formal Methods: Toward Safe Control Through Proof and Learning. In Proceedings of the Thirty-Second AAAI Conference on Artificial Intelligence, (AAAI-18), the 30th innovative Applications of Artificial Intelligence (IAAI-18), and the 8th AAAI Symposium on Educational Advances in Artificial Intelligence (EAAI-18), New Orleans, Louisiana, USA, February 2-7, 2018, Sheila A. McIlraith and Kilian Q. Weinberger (Eds.). AAAI Press, 6485-6492. https://www.aaai.org/ocs/index.php/ AAAI/AAAI18/paper/view/17376

[22] Teuvo Kohonen. 2013. Essentials of the self-organizing map. Neural Networks 37 (2013), 52-65. https://doi.org/10.1016/j.neunet.2012.09.018

[23] Christine Milchram, Geerten Van de Kaa, Neelke Doorn, and Rolf Künneke. 2018. Moral values as factors for social acceptance of smart grid technologies. Sustainability 10, 8 (2018), 2703.

[24] James H Moor. 2006. The nature, importance, and difficulty of machine ethics. IEEE intelligent systems 21, 4 (2006), 18-21.

[25] Shelley Nason and John E Laird. 2005. Soar-RL: Integrating reinforcement learning with Soar. Cognitive Systems Research 6, 1 (2005), 51-59.

[26] Anand S. Rao. 1996. AgentSpeak(L): BDI Agents Speak Out in a Logical Computable Language. In Agents Breaking Away, 7th European Workshop on Modelling Autonomous Agents in a Multi-Agent World, Eindhoven, The Netherlands, Fanuary 22-25, 1996, Proceedings (Lecture Notes in Computer Science, Vol. 1038), Walter Van de Velde and John W. Perram (Eds.). Springer, 42-55. https://doi.org/10.1007/BFb0031845
[27] Nicolas P. Rougier and Yann Boniface. 2011. Dynamic self-organising map. Neurocomputing 74, 11 (2011), 1840-1847. https://doi.org/10.1016/j.neucom.2010. 06.034

[28] Daniel Schiff, Justin Biddle, Jason Borenstein, and Kelly Laas. 2020. What's Next for AI Ethics, Policy, and Governance? A Global Overview. In AIES '20: AAAI/ACM Conference on AI, Ethics, and Society, New York, NY, USA, February 7-8, 2020, Annette N. Markham, Julia Powles, Toby Walsh, and Anne L. Washington (Eds.). ACM, 153-158. https://doi.org/10.1145/3375627.3375804

[29] Richard S. Sutton and Andrew G. Barto. 1998. Reinforcement Learning: An Introduction. IEEE Trans. Neural Networks 9, 5 (1998), 1054-1054. https://doi. org/10.1109/TNN.1998.712192

[30] Judith Jarvis Thomson. 1976. Killing, letting die, and the trolley problem. The Monist 59, 2 (1976), 204-217.

[31] Francisco J Varela. 1999. Ethical know-how: Action, wisdom, and cognition. Stanford University Press.

[32] Christopher J. C. H. Watkins and Peter Dayan. 1992. Q-Learning. Machine Learning 8, 3 (May 1992), 279-292. https://doi.org/10.1007/BF00992698

[33] Yueh-Hua Wu and Shou-De Lin. 2018. A Low-Cost Ethics Shaping Approach for Designing Reinforcement Learning Agents. In Proceedings of the Thirty-Second $A A A I$ Conference on Artificial Intelligence, (AAAI-18), the 30th innovative Applications of Artificial Intelligence (IAAI-18), and the 8th AAAI Symposium on Educational Advances in Artificial Intelligence (EAAI-18), New Orleans, Louisiana, USA, February 2-7, 2018, Sheila A. McIlraith and Kilian Q. Weinberger (Eds.). AAAI Press, 1687-1694. https://www.aaai.org/ocs/index.php/AAAI/AAAI18/ paper/view/16195

[34] Kexin Yi, Chuang Gan, Yunzhu Li, Pushmeet Kohli, Jiajun Wu, Antonio Torralba, and Joshua B. Tenenbaum. 2020. CLEVRER: CoLlision Events for Video REpresentation and Reasoning. arXiv:1910.01442 [cs.CV]

[35] Han Yu, Zhiqi Shen, Chunyan Miao, Cyril Leung, Victor R. Lesser, and Oiang Yang. 2018. Building Ethics into Artificial Intelligence. In Proceedings of the 27th International foint Conference on Artificial Intelligence (IFCAI'18). AAAI Press, Stockholm, Sweden, 5527-5533. 